\title{
HIF1a overexpression enhances diabetic wound closure in high glucose and low oxygen conditions by promoting adipose- derived stem cell paracrine function and survival
}

\author{
Jin Xu', Xiaoyu Liu' ${ }^{2}$ Feng Zhao ${ }^{3}$, Ying Zhang ${ }^{4}$ and Zhe Wang ${ }^{4^{*}}$
}

\begin{abstract}
Background: Adipose-derived stem cell (ADSC) transplantation is a promising strategy to promote wound healing because of the paracrine function of stem cells. However, glucose-associated effects on stem cell paracrine function and survival contribute to impaired wound closure in patients with diabetes, limiting the efficacy of ADSC transplantation. Hypoxia-inducible factor (HIF)1 a plays important roles in wound healing, and in this study, we investigated the effects of HIF1a overexpression on ADSCs in high glucose and low oxygen conditions.

Methods: Adipose samples were obtained from BALB/C mice, and ADSCs were cultured in vitro by digestion. Control and HIF1a-overexpressing ADSCs were induced by transduction. The mRNA and protein levels of angiogenic growth factors in control and HIF1a-overexpressing ADSCs under high glucose and low oxygen conditions were analyzed by quantitative reverse transcription-polymerase chain reaction and western blotting. The effects of ADSC HIF1a overexpression on the proliferation and migration of mouse aortic endothelial cells (MAECs) under high glucose were evaluated using an in vitro coculture model. Intracellular reactive oxygen species (ROS) and 8-hydroxydeoxyguanosine (8-OHdG) levels in ADSCs were observed using 2,7-dichlorodihydrofluorescein diacetate staining and enzyme-linked immunosorbent assays, respectively. Apoptosis and cell cycle analysis assays were performed by flow cytometry. An in vivo full-thickness skin defect mouse model was used to evaluate the effects of transplanted ADSCs on diabetic wound closure.

Results: In vitro, HIF1a overexpression in ADSCs significantly increased the expression of vascular endothelial growth factor A, fibroblast growth factor 2, and C-X-C motif chemokine ligand 12, which were inhibited by high glucose. HIF1a overexpression in ADSCs alleviated high glucose-induced defects in MAEC proliferation and migration and significantly suppressed ADSC ROS and 8-OHdG levels, thereby decreasing apoptosis and enhancing survival. In vivo, HIF1a overexpression in ADSCs prior to transplantation significantly enhanced angiogenic growth factor expression, promoting wound closure in diabetic mice.

(Continued on next page)
\end{abstract}

\footnotetext{
* Correspondence: wangz@sj-hospital.org

${ }^{4}$ Department of Pathology, Shengjing Hospital of China Medical University,

No. 36 Sanhao Street, Heping District, Shenyang 110004, China

Full list of author information is available at the end of the article
}

(c) The Author(s). 2020 Open Access This article is licensed under a Creative Commons Attribution 4.0 International License, which permits use, sharing, adaptation, distribution and reproduction in any medium or format, as long as you give appropriate credit to the original author(s) and the source, provide a link to the Creative Commons licence, and indicate if changes were made. The images or other third party material in this article are included in the article's Creative Commons licence, unless indicated otherwise in a credit line to the material. If material is not included in the article's Creative Commons licence and your intended use is not permitted by statutory regulation or exceeds the permitted use, you will need to obtain permission directly from the copyright holder. To view a copy of this licence, visit http://creativecommons.org/licenses/by/4.0/. The Creative Commons Public Domain Dedication waiver (http://creativecommons.org/publicdomain/zero/1.0/) applies to the data made available in this article, unless otherwise stated in a credit line to the data. 
(Continued from previous page)

Conclusions: HIF1a overexpression in ADSCs efficiently alleviates high glucose-induced paracrine dysfunction, decreases oxidative stress and subsequent DNA damage, improves viability, and enhances the therapeutic effects of ADSCs on diabetic wound healing.

Keywords: Hypoxia-inducible factor 1a, Adipose-derived stem cells, Wound healing, High glucose, Reactive oxygen species, Mice, Stem cell transplantation

\section{Background}

Diabetes mellitus is a serious chronic metabolic disorder characterized by high glucose levels, which induces oxidative stress. It affects more than 400 million people worldwide, with a predicted $>50 \%$ increase in cases by 2030 [1-3]. Micro- and macrovascular diseases are key complications of diabetes and may be important risk factors for death in diabetic patients [4, 5]. Endothelial dysfunction is strongly associated with the risk of vascular disease and results in impaired wound healing [5-7]. Previous studies have shown that a high glucose concentration downregulates the expression of various angiogenic growth factors, such as vascular endothelial growth factor A (VEGFA), fibroblast growth factor 2 (FGF2), and C-X-C motif chemokine ligand 12 (CXCL12), which in turn suppress endothelial cell (EC) functions, leading to impaired angiogenesis and delayed wound closure [8].

Adipose-derived stem cells (ADSCs) support the engineering of functional tissue by secreting angiogenic and cytoprotective factors, which act in a paracrine fashion to promote vascularization and cell survival [9]. Due to this paracrine function, ADSCs are being increasingly investigated as cellular therapies for different diseases, as well as for burns and trauma. However, diabetes impairs the paracrine function and survival of transplanted stem cells [10]. Previous in vitro studies have shown that high glucose induces reactive oxygen species (ROS) production and subsequent ADSC apoptosis [11]. Therefore, an effective method to improve stem cell paracrine function and survival in the diabetic microenvironment is urgently needed [12].

Hypoxia-inducible factor 1 (HIF1) is a master regulator of oxygen homeostasis and an important determinant of healing outcomes. HIF1 contributes to all stages of wound healing through its roles in migration, survival under hypoxia, cell division, growth factor release, and matrix synthesis. HIF $1 \alpha$ is the regulatory subunit of HIF1 [13]. Positive regulators of HIF1 $\alpha$, such as prolyl4-hydroxylase inhibitors, enhance diabetic ischemic wound closure and are currently undergoing clinical trials for the treatment of several human ischemic conditions [14]. Specifically, HIF1 $\alpha$ enhances VEGFA expression by directly binding its promoter [15]. However, the protective effects of ADSC HIF1 $\alpha$ on diabetic wound closure have not been reported.
In this study, we examined whether HIF1 $\alpha$ overexpression in ADSCs improves diabetic wound closure in mice and investigated the possible mechanisms involved. These data reveal an effective strategy to enhance wound healing treatment under diabetic conditions.

\section{Materials and methods}

\section{Ethical considerations}

This study was approved by the Laboratory Animal Welfare and Ethics Committee of China Medical University and conducted according to the Guide for the Care and Use of Laboratory Animals.

\section{Isolation and treatment of mouse ADSCs}

Twenty BALB/c mice (male; body weight 18-24 g; 8 weeks old) were purchased from Beijing Huafukang Bioscience Co. Inc. (Beijing, China). ADSCs were isolated from the inguinal fat pad as previously described [16]. Cells were suspended in Dulbecco's modified Eagle's medium (DMEM) supplemented with $10 \%$ fetal bovine serum, $100 \mathrm{U} / \mathrm{mL}$ penicillin, and $100 \mu \mathrm{g} / \mathrm{mL}$ streptomycin. The cultures were maintained at $37^{\circ} \mathrm{C}$ in a humidified atmosphere containing 5\% $\mathrm{CO}_{2}$. ADSCs were used in experiments after 3-6 passages. To overexpress HIF1 $\alpha$, ADSCs were transduced with either an empty lentivirus (as a control) or a lentivirus expressing recombinant HIF1 $\alpha$ using a cytomegalovirus promoter (Origene, Shanghai, China) for $48 \mathrm{~h}$. For high glucose experiments, cells were cultured in serum-free DMEM containing high glucose $(25 \mathrm{mM})$ for $24 \mathrm{~h}$ prior to lentiviral transduction. Cells cultured with $5.5 \mathrm{mM}$ glucose were used as normoglycemic controls. After $48 \mathrm{~h}$, cells were harvested to detect mRNA and protein expression. All experiments were performed in a hypoxia chamber (Mitsubishi GAS Chemical, Tokyo, Japan; oxygen concentration: $0.1 \%$ ) as previously described [17]. For ADSC transplantation experiments, donor ADSCs transduced with empty or HIF1 $\alpha$-encoding lentiviruses were subcutaneously injected at the time of skin wound induction.

\section{Quantitative reverse transcription-polymerase chain reaction (RT-qPCR)}

VEGFA, HIF1 $\alpha$, FGF2, and CXCL12 mRNA levels in ADSCs or tissues were examined by RT-qPCR as 
previously reported [17]. PCR primer sequences are listed in Table 1. CXCL12 primers (QT00161112) were purchased from Qiagen (Valencia, CA, USA). Briefly, after reverse transcription, qPCR was performed using the iQ5 real-time PCR detection system (Bio-Rad, Hercules, CA, USA) with SYBR Supermix (Qiagen). The fold change in the expression levels of each target mRNA under experimental and control conditions was calculated using the $2^{-\Delta \Delta C T}$ method [18], relative to glyceraldehyde 3-phosphate dehydrogenase (GAPDH).

\section{Western blotting}

VEGFA, HIF1 $\alpha$, FGF2, and CXCL12 protein levels in ADSCs and tissues were determined by western blotting as previously described [11, 12]. Briefly, after electrophoresis and transfer to polyvinylidene difluoride membranes, proteins were incubated with antibodies against HIF1 $\alpha$ (ab216842, 1:500, Abcam, Cambridge, MA, USA), anti-VEGFA (ab1316, 1:100, Abcam), FGF2 (ab92337; 1: 300; Abcam), CXCL12 (ab137867; 1:300; Abcam), and GAPDH (\#5174; 1:20,000; Cell Signaling Technologies, Danvers, MA, USA) at $4{ }^{\circ} \mathrm{C}$ overnight. Proteins were visualized using an enhanced chemiluminescence detection system (Bio-Rad, Hercules, CA, USA) and exposure to X-ray film and quantified by laser scanning densitometry (GE Healthcare Life Sciences, Piscataway, NJ). GAPDH was used as a loading control.

\section{ADSC-MAEC coculture}

MAECs were purchased from CHI Scientific (Maynard, MA, USA) and cultured in endothelial basal medium (CHI Scientific) at $37^{\circ} \mathrm{C}$ in a humidified incubator with 95\% $\mathrm{O}_{2}$ and $5 \% \mathrm{CO}_{2}$. To examine the effects of ADSCs on MAEC proliferation and migration, MAECs were seeded at $0.5 \times 10^{4}$ cells $/ \mathrm{cm}^{2}$ in 24 -well plates and grown to $80-90 \%$ confluence, then grown in serum-free medium for $12 \mathrm{~h}$ prior to coculture. For high glucose experiments, control or HIF1 $\alpha$-overexpressing ADSCs were seeded at $0.5 \times 10^{4}$ cells $/ \mathrm{cm}^{2}$ in CoStar Transwell cell culture inserts $(0.4 \mu \mathrm{m}$ pore size; Corning, Corning, NY, USA) and cultured in serum-free DMEM containing high glucose $(25 \mathrm{mM})$ for $24 \mathrm{~h}$ prior to coculture. For vascular endothelial growth factor receptor 2 (VEGFR2)

Table 1 Primers used in RT-qPCR analysis

\begin{tabular}{lll}
\hline Transcript & Primer (5'-3') & Product length \\
\hline HIF1a & Forward: CAAGAAACCACCCATGAC & $165 \mathrm{bp}$ \\
& Reverse: GGCTCATAACCCATCAAC & \\
VEGFA & Forward: AGGGCAGAATCATCACGAAGT & $75 \mathrm{bp}$ \\
& Reverse: AGGGTCTCGATTGGATGGCA & \\
FGF2 & Forward: GGGACTGGTCAGTATTAGAGGT & $215 \mathrm{bp}$ \\
& Reverse: CTCTGGAGTCCGTCTTTTT & \\
GAPDH & Forward: CTGCCCAGAACATCATCC & $197 \mathrm{bp}$ \\
& Reverse: CAGATGCCTGCTTCAC & \\
\hline
\end{tabular}

inhibitor (ZD6474) experiments, MAECs were pretreated with ZD6474 for $24 \mathrm{~h}$ prior to coculture with ADSCs. In group 1 (High glucose), control ADSCs were cocultured with MAECs; in group 2 (High+HIF1 $\alpha$ ), HIF1 $\alpha$-overexpressing ADSCs were cocultured with MAECs; in group 3 (High+HIF1 $\alpha+$ ZD6474), HIF1 $\alpha-$ overexpressing ADSCs were cocultured with MAECs pre-treated with ZD6474; and in group 4 (High+ZD6474), control ADSCs were cocultured with MAECs pre-treated with ZD6474. For MAEC proliferation and apoptosis assays, MAECs were harvested after 2 and $3 \mathrm{~d}$ of coculture with ADSCs, respectively.

\section{Cell proliferation assays}

The 3-(4, 5-dimethylthiazol-2-yl)-2, 5-diphenyltetrazolium bromide (MTT) assay (Andwin Scientific, Addison, IL, USA) was used to assess relative cell growth and viability according to the manufacturer's instructions. ADSCs or MAECs $\left(5 \times 10^{3}\right)$ were seeded into 96-well culture plates for $24 \mathrm{~h}$ before incubation with MTT for $4 \mathrm{~h}$ at $37^{\circ} \mathrm{C}$. The absorbance of the medium was measured at $540 \mathrm{~nm}$, and the data are expressed as ratios of the control value. Three independent experiments were conducted, with two technical replicates per experiment.

\section{Apoptosis assays}

Apoptotic cells were quantified using the Annexin VFITC/PI Apoptosis Detection Kit (KeyGen Biotech, Jiangsu, China) according to the manufacturer's instructions. Briefly, cells were trypsinized and resuspended in binding buffer containing Annexin V-fluorescein isothiocyanate (FITC) and propidium iodide (PI) at $22-24{ }^{\circ} \mathrm{C}$ for $10 \mathrm{~min}$ before analysis on a FACSCalibur flow cytometer (BD Biosciences, San Jose, CA). Three independent experiments were conducted, with two technical replicates per experiment.

\section{Transwell migration assays}

MAEC migration was measured using Transwell migration assays $(8.0 \mu \mathrm{m}$ pore size; Corning-Costar) according to the manufacturer's protocol. In brief, ADSCs were seeded in the bottom chamber of 24-well plates at $0.5 \times$ $10^{4}$ cells $/ \mathrm{cm}^{2}$. A cell culture insert with $8.0 \mu \mathrm{m}$ pores was placed in the well, and MAECs were added at $0.5 \times$ $10^{4}$ cells $/ \mathrm{cm}^{2}$ and incubated at $37^{\circ} \mathrm{C}$ in $5 \% \mathrm{CO}_{2}$ for $24 \mathrm{~h}$. Following incubation, the filters were removed and cells remaining on the upper surface of the membrane were removed with a cotton swab. The membranes were washed with PBS two times, and cells adhering beneath the membrane were fixed in $4 \%$ paraformaldehyde and stained with 4',6-diamidino-2-phenylindole [19]. The number of migrated cells was quantified by counting eight randomly selected regions under a fluorescence microscope (Leica Wetzlar, Germany). 


\section{In vitro wound healing assays}

We also used scratch assays to estimate the migration ability of MAECs in vitro. Briefly, MAECs were seeded in the bottom chamber of 24-well plates at $0.5 \times$ $10^{4} \mathrm{cells} / \mathrm{cm}^{2}$ and grown to $80-90 \%$ confluence, then grown in serum-free medium for $12 \mathrm{~h}$. Then, the confluent layer of cells was scratched using a sterile $200 \mu \mathrm{L}$ pipette tip. After washing with PBS, MAECs were cocultured with ADSCs. Images were recorded immediately after the monolayers were scratched $(0 \mathrm{~h})$ and $24 \mathrm{~h}$ later. Scratched areas were measured using Image-Pro Plus 6.0 software. The results are presented as the percentage of wound closure, which was calculated as follows: [wound area (initial) - wound area(final)]/wound area (initial) $\times$ $100[10,15]$. Three independent experiments were conducted, with two technical replicates per experiment.

\section{ROS measurements}

Intracellular ROS levels were assessed using the peroxide-sensitive fluorescent probe 2,7-dichlorodihydrofluorescein diacetate (DCFH-DA, Beyotime Institute of Technology, Jiangsu, China) [20]. ADSCs were cultured as described and subsequently exposed to $10 \mathrm{~mol} /$ L DCFH-DA for $30 \mathrm{~min}$ at $37^{\circ} \mathrm{C}$. Fluorescence intensity was observed in a DMI6000B inverted microscope (Leica, Wetzlar, Germany). Three independent experiments were conducted, with two technical replicates per experiment.

\section{Enzyme-linked immunosorbent assays (ELISAs)}

To detect DNA damage, 8-hydroxydeoxyguanosine (8OHdG) was detected using the 8-OHdG ELISA kit (BYE10099; Shanghai Bangyi Biotechnology, Shanghai, China) according to the manufacturer's instructions. ADSCs were washed twice with PBS, lysed in $100 \mu \mathrm{L}$ radioimmunoprecipitation buffer (Beyotime Institute of Technology), and centrifuged at $3000 \times g$ for $20 \mathrm{~min}$ at $4{ }^{\circ} \mathrm{C}$. Each sample $(10 \mu \mathrm{L})$ was combined with $40 \mu \mathrm{L}$ sample diluent and added to the enzyme-labeled plate before sealing and incubation at $37^{\circ} \mathrm{C}$ for $30 \mathrm{~min}$. After incubation, the absorbance was measured at $450 \mathrm{~nm}$. Three independent experiments were conducted, with two technical replicates per experiment.

\section{Cell cycle analysis}

ADSCs were detached from plates, centrifuged, and resuspended, washed with PBS, and fixed in $70 \%$ ethanol at $4{ }^{\circ} \mathrm{C}$ for $24 \mathrm{~h}$. Cells were then suspended in $300 \mu \mathrm{L}$ dyeing solution $(500 \mu \mathrm{L}$ buffer containing $10 \mu \mathrm{L}$ RNase A and $25 \mu \mathrm{L}$ PI) and incubated at $37^{\circ} \mathrm{C}$ for $30 \mathrm{~min}$ in the dark. Cells $\left(1 \times 10^{4}\right)$ were subjected to cell cycle analysis on a FACSCalibur flow cytometer (BD Biosciences). Data are from three independent experiments were conducted, with two technical replicates per experiment.

\section{ADSC labelling}

To track ADSCs in vivo, control- and HIF1 $\alpha$-transduced ADSCs were grown to confluence in cell culture dishes and on glass slides and treated with $20-30 \mu \mathrm{g} / \mathrm{mL}$ superparamagnetic polyethyleneimine-coated iron oxide nanoparticles (SPIONs) (Nanjing Nanoeast Biotech, Nanjing, China) for $24 \mathrm{~h}$ [21].

\section{Diabetic wound closure model}

To evaluate the therapeutic effects of HIF $1 \alpha-$ overexpressing ADSCs on diabetic wound closure in vivo, a mouse model was established. Briefly, following fur removal from the dorsal surface, the mice were anesthetized with sodium pentobarbital $(0.5 \mathrm{mg} / \mathrm{g})$, and a $10-\mathrm{mm}$ full-thickness round excisional skin wound was made on the back of each mouse with a sterile $10-\mathrm{mm}$ punch biopsy tool. BALB/c mice (male; body weight 18 $24 \mathrm{~g} ; 8$ weeks old) were randomly divided into three groups ( $n=12$ mice/group). In all three groups, diabetes was induced by intraperitoneal (i.p.) injection of $150 \mathrm{mg} /$ $\mathrm{kg}$ body weight streptozotocin (STZ). Mice with blood glucose levels $\geq 16.7 \mathrm{mmol} / \mathrm{L}$ were considered diabetic. One week later, 36 wounds were generated. Group 1 (high glucose) mice were left untreated, group 2 (high+ADSCs) mice received control ADSCs, and group 3 $($ high + ADSCs-HIF1 $\alpha)$ mice received HIF1 $\alpha$ overexpressing ADSCs. ADSCs were incubated with SPIONs for in vivo cell tracking using Prussian blue staining. Control and HIF1 $\alpha$-overexpressing ADSCs $(1 \times$ $10^{6}$ in $60 \mu \mathrm{L}$ PBS) were subcutaneously injected with a 25 -gauge needle at the time of skin wound induction, and wounds were monitored for 2 weeks.

\section{Statistical analysis}

Data from repeated experiments are presented as the mean \pm standard deviation (SD). Data were compared between groups using one- or two-way analysis of variance (ANOVA). Following ANOVA, the least significant difference post hoc test or Dunnett's $t$ test with Bonferroni correction was used to analyze the significance of differences between the mean values of the experimental and control groups. $P<0.05$ was considered significant.

\section{Results}

HIF1a overexpression promotes ADSC paracrine function

To evaluate the effects of HIF1 $\alpha$ on diabetic wound closure, we isolated ADSCs and transduced them with control or lentiviruses expressing either null (as a control) or recombinant HIF1 $\alpha$ under a CMV promoter. HIF1 $\alpha$ mRNA levels in the transduced cells were determined by RT-qPCR (Fig. 1a). While high glucose and low oxygen disrupted VEGFA, FGF2, and CXCL12 expression, HIF1 $\alpha$ overexpression in ADSCs restored their expression at both the mRNA (Fig. 1b) and protein (Fig. 1c, d) 


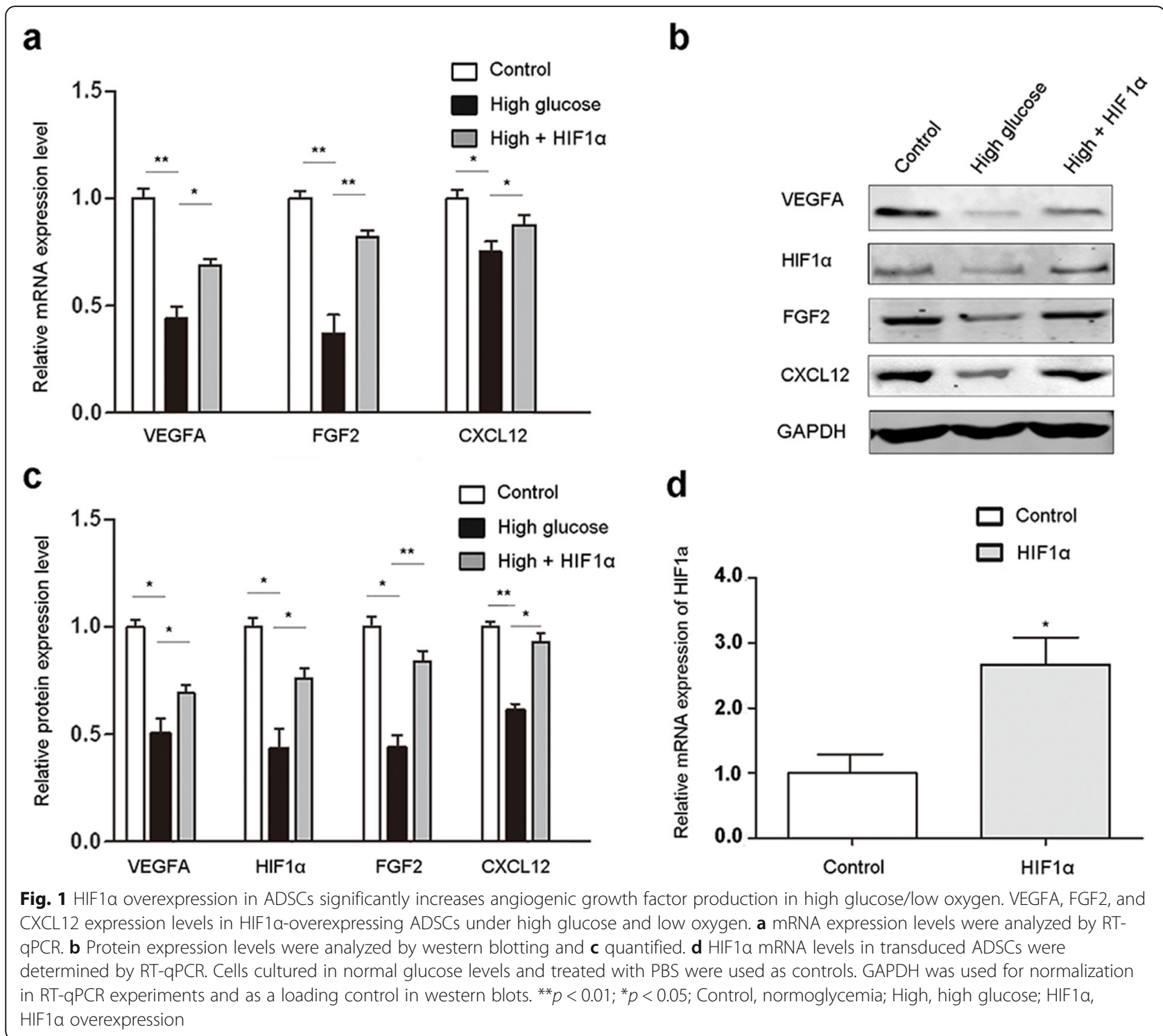

levels. FGF2 and CXCL12 promote cell migration, while VEGFA promotes cell survival during oxidative stress. These results demonstrate that HIF1 $\alpha$ overexpression in ADSCs enhances their paracrine function in high glucose/low oxygen conditions.

\section{HIF1a overexpression in ADSCs alleviates high glucose- induced MAEC dysfunction}

EC dysfunction under high glucose has been shown to impair wound healing [7]. Therefore, we established a coculture model to explore the effects of HIF1 $\alpha$-overexpressing ADSCs on MAEC function in a high glucose environment. Proliferation and migration are crucial EC functions, and impairment of these processes can prevent angiogenesis in the wound area and delay wound healing [7, 22]. In MAECs, high glucose decreased proliferation (Fig. 2a), increased apoptosis (Fig. 2b), and decreased migration (Fig. 2c-f), while HIF1 $\alpha$-overexpressing ADSCs restored these functions.

We next investigated whether HIF1 $\alpha$ overexpression in ADSCs alleviates high glucose-induced MAEC dysfunction by regulating VEGFR2 expression. We analyzed the proliferation, apoptosis, and migration of MAECs treated with both HIF1 $\alpha$ overexpressing-ADSCs and a VEGFR2 inhibitor (ZD6474) in high glucose. ZD6474 treatment decreased proliferation (Fig. 3a), increased apoptosis (Fig. 3b), and decreased the migration potential (Fig. 3c-f) of MAECs. These results demonstrate that VEGFR2 inhibition nullifies the effects of ADSC HIF1 $\alpha$ overexpression on MAECs in high glucose, indicating that HIF1 $\alpha$ overexpression in ADSCs alleviates MAEC dysfunction in high glucose through VEGFR2 signaling. 


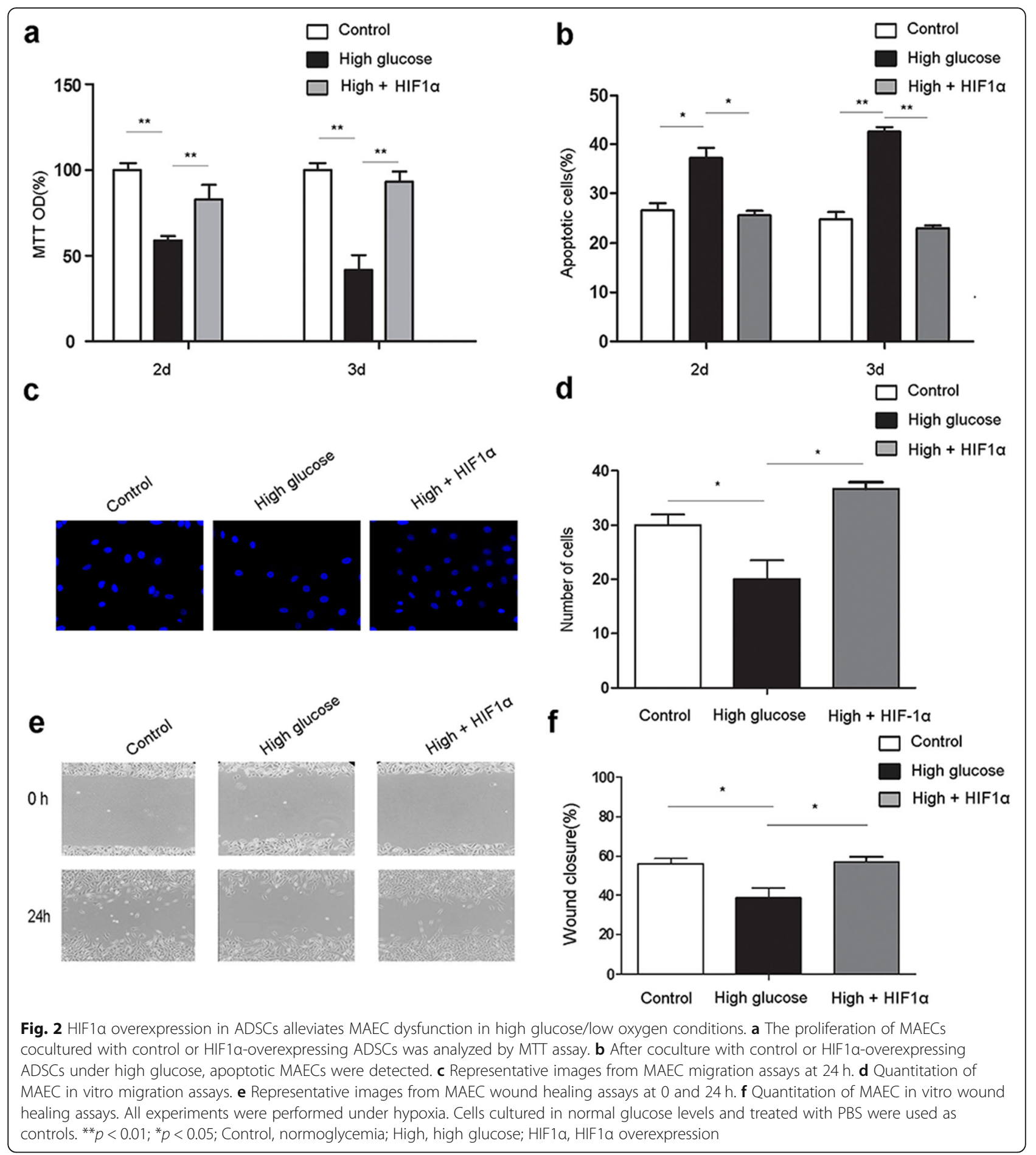

HIF1a overexpression promotes ADSC survival in high glucose/low oxygen conditions

The survival of transplanted ADSCs is the most critical factor for successful ADSC-based therapy [22, 23]. However, high glucose levels due to diabetes induce oxidative stress that damages cells and suppresses their survival [11]. Thus, we examined whether HIF1 $\alpha$ overexpression in ADSCs could suppress ROS generation under high glucose. When cells were transduced with HIF1 $\alpha$ prior to exposure to high glucose, HIF1 $\alpha$ overexpression significantly reduced intracellular ROS levels (Fig. 4a). Next, 8-OHdG levels were assessed to 


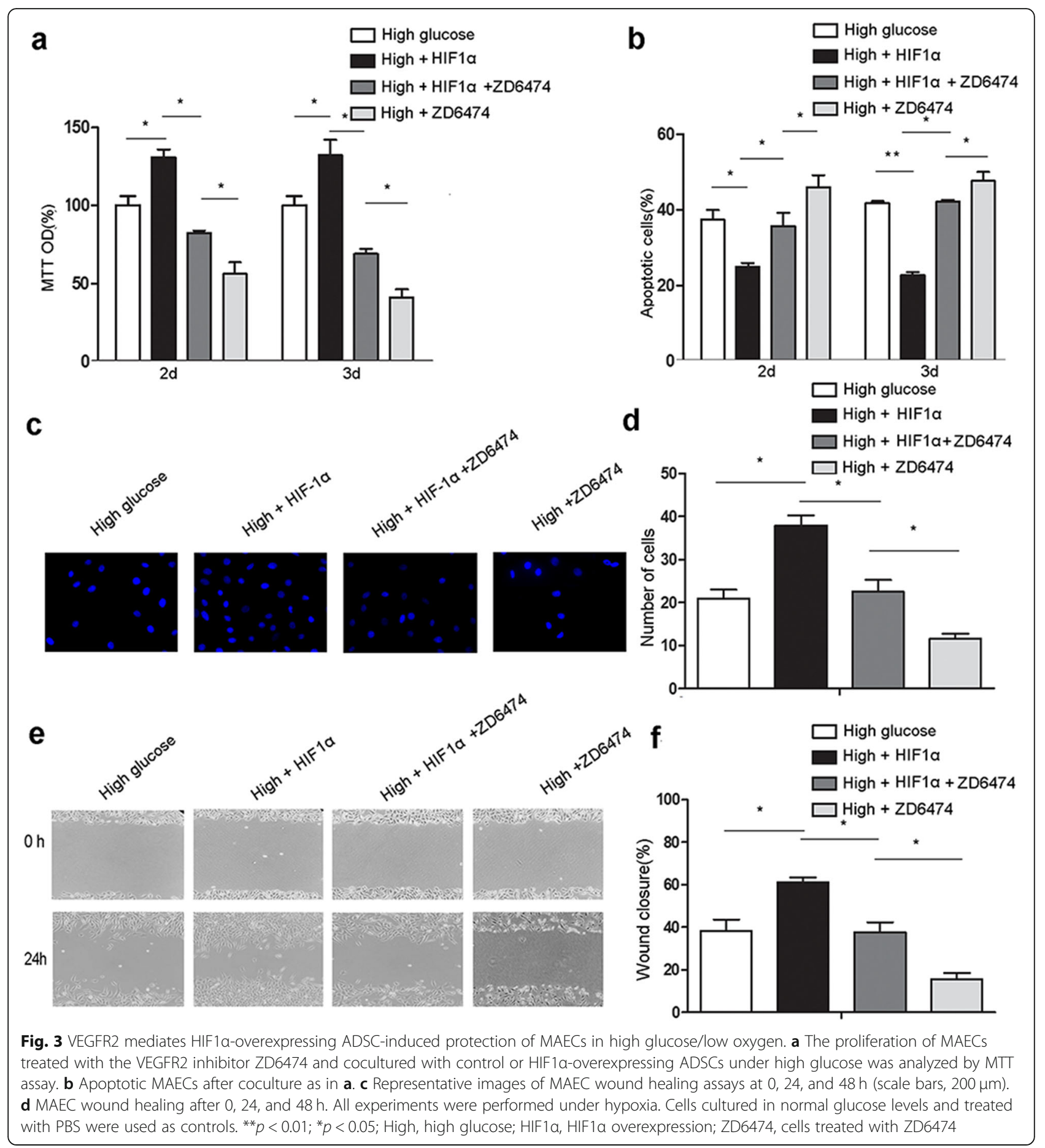

examine the extent of DNA damage caused by oxidation. High glucose treatment significantly increased 8OHdG levels compared with control conditions, and HIF1 $\alpha$ overexpression significantly decreased ADSC 8OHdG levels compared with control ADSCs (Fig. 4b). Concomitantly, HIF1 $\alpha$ overexpression restored the proliferative potential of the ADSCs, which was suppressed by high glucose (Fig. 4c).
Next, we analyzed the effect of HIF1 $\alpha$ overexpression on the ADSC apoptosis rate in high glucose. High glucose resulted in increased ADSC apoptosis, which was significantly reduced by HIF1 $\alpha$ overexpression (Fig. 4d). Cell cycle analysis revealed that the percentage of cells in G2/M phase significantly increased with high glucose compared to control conditions, and HIF1 $\alpha$ overexpression in ADSCs 


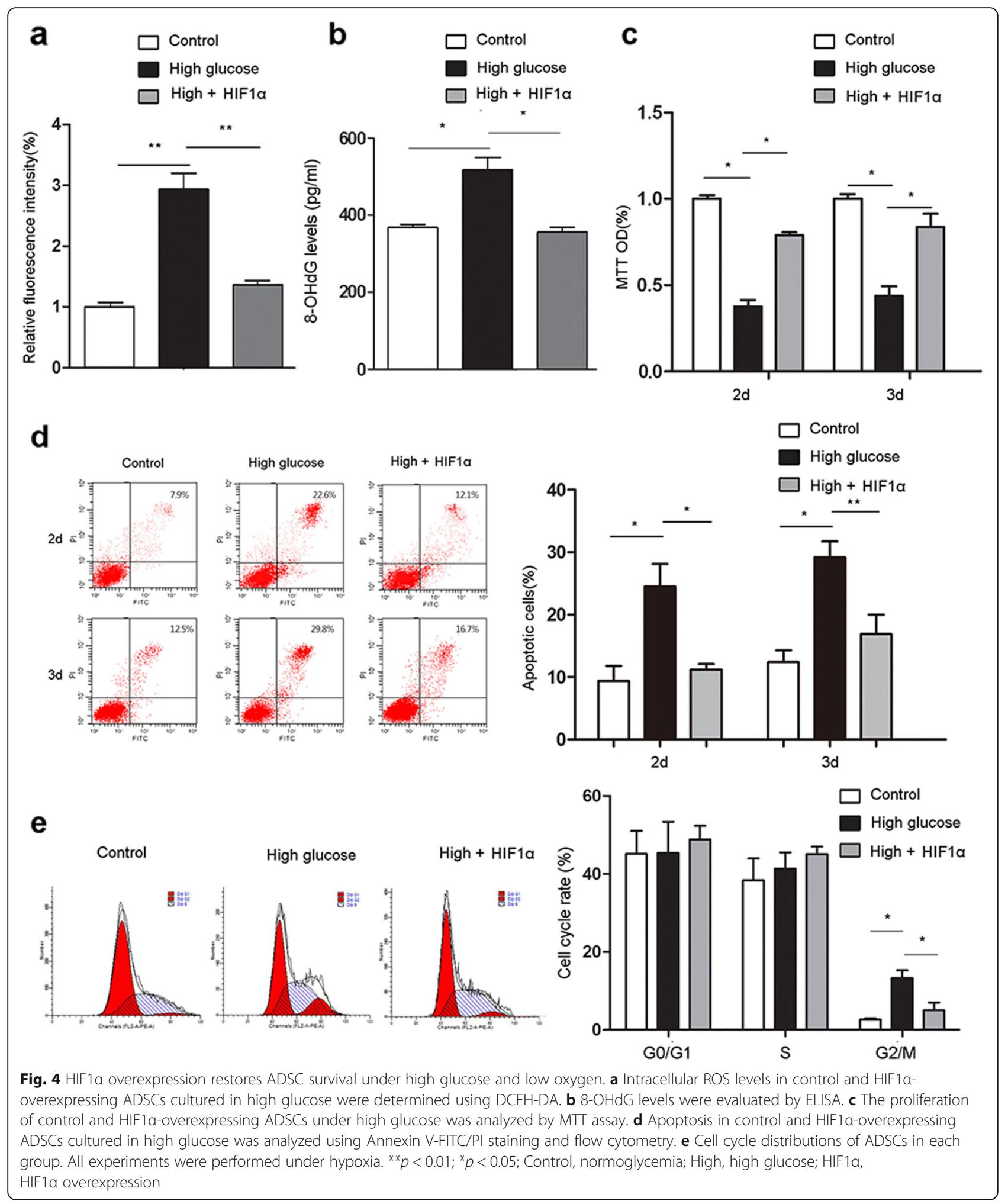

significantly decreased G2/M phase cells compared with high glucose (Fig. 4e); conversely, no significant changes in the G0/G1 or $\mathrm{S}$ phases were observed. Together, these results demonstrate that HIF1 $\alpha$ overexpression in ADSCs may repress ROS generation, prevent DNA damage, improve cell cycle arrest, and promote ADSC proliferation and survival in diabetic environments. 


\section{HIF1a overexpression enhances diabetic wound closure by promoting the paracrine function of ADSCs}

To assess the therapeutic effects of HIF1 $\alpha$ overexpressing ADSCs on diabetic wound healing, we established a mouse model of diabetic wound healing and transplanted the wounds with control or HIF1 $\alpha$ overexpressing ADSCs (Fig. 5a). The in vivo wound repair ability of ADSCs was examined by assessing wound closure rates. As presented in Fig. 5b and c, on the 7th day, there was a small but insignificant increase in the healing rate in the ADSC transplantation group compared to the control group. However, on the 14th day, both control and HIF1 $\alpha$-overexpressing ADSCs exhibited positive effects on wound closure, and HIF1 $\alpha$ overexpressing ADSCs significantly promoted wound closure compared to control ADSCs (Fig. 5b, c). To visualize migrated $\mathrm{ADSCs}$ in the wounds, they were labeled with SPIONs and sectioned tissues were stained with Prussian blue (Fig. 5d). VEGFA, FGF2, and CXCL12 protein and mRNA expression levels were upregulated at the wound sites of mice transplanted with HIF1 $\alpha$-overexpressing ADSCs (Fig. 5e-g). Taken together, these results demonstrate for the first time that HIF1 $\alpha$ overexpression in ADSCs promotes their paracrine function and survival by decreasing ROS production, thereby enhancing their therapeutic effects in diabetic wound healing (Fig. 6).

\section{Discussion}

Diabetes affects $>9 \%$ of adults worldwide [21]. People with type 2 diabetes mellitus (T2DM) have a 25-fold higher risk of limb loss than nondiabetics, mainly due to impaired wound healing. ADSC transplantation has been used in regenerative medicine as a cellular treatment for many diseases [9, 24]. However, high glucose affects the expression of various ADSC factors, resulting in systematic impairment of their physiological pathways and paracrine function and decreased survival after transplantation [22]. High glucose and advanced glycation end products severely disrupt ADSC survival, most likely due to excessive ROS production [11, 12]. Therefore, an effective method of improving stem cell paracrine function and survival in diabetic microenvironments is urgently needed.

The physiologic response to local wound hypoxia plays a critical role in determining the success of the normal healing process. HIF1 $\alpha$, a master regulator of oxygen homeostasis, is an important determinant of healing outcomes that contributes to all stages of wound healing [25]. HIF1 deficiency and subsequent failure to respond to hypoxic stimuli lead to chronic hypoxia, which contributes to the formation of nonhealing diabetic ulcers [26]. A recent report demonstrated the protective mechanism of HIF1 $\alpha$ overexpression in reinfused blood cells in enhancing diabetic ischemic wound closure [27].
Although positive regulators of HIF1 are currently in clinical trials for the treatment of several human ischemic conditions, it has been reported that HIF1 $\alpha$ stabilization in ischemic wounds leads to impaired reepithelialization and delayed wound closure, and HIF1 $\alpha$ overexpression can contribute to fibrosis and excessive scarring [28-30]. A recent study showed that HIF1 $\alpha$ deletion facilitated adipose stem cell-mediated repair of renal fibrosis in diabetic mice [31]. Diabetic wound healing is a complicated pathological process occurring in a complex internal environment that features high glucose, hypoxia, and oxidative stress. However, the effects of HIF1 $\alpha$ overexpressing ADSCs on diabetic wound healing have not been reported. We examined whether HIF1 $\alpha$ overexpression in ADSCs can improve their paracrine function and survival in a diabetic environment. A previous report showed that VEGFA, FGF2, and CXCL12 are major components of the stem cell secretome, which is essential for angiogenesis during wound healing [9], and our results demonstrate that HIF1 $\alpha$-overexpressing ADSCs robustly enhance the expression of these factors, which are crucial for cell survival and wound healing in high glucose. Endothelial dysfunction is an important contributor to compromised wound healing in this context, which manifests in diabetes, atherosclerosis, fibrosis, and vascular occlusion. ECs display high sensitivity to radiation injury, yet these cells play an essential role in wound healing. ADSCs secrete various cytokines and growth factors to support the functions of other cells, including their growth and migration [25], and media derived from hypoxic preconditioned ADSCs can support EC survival and endothelial tube formation in vitro [26]. We established a coculture model to examine if HIF1 $\alpha$ overexpression in ADSCs prevents EC dysfunction in high glucose. MAEC proliferation, survival, and migration in high glucose were significantly improved when cocultured with HIF1 $\alpha$-overexpressing ADSCs compared with control ADSCs. This suggests that ADSCs compensate for endothelial dysfunction in diabetic microenvironments by restoring growth factor expression.

VEGF isoforms belong to the platelet-derived growth factor family and play central roles in the regulation of angiogenesis. VEGFR2 exhibits strong tyrosine kinase activity in response to proangiogenic signals and regulates EC proliferation, migration, vascular permeability, and secretion, making it a critical factor in EC function [32]. A high glucose-induced reduction in VEGFR2 cell surface abundance in diabetic mice was reversed by treatment with the antioxidant $N$-acetyl-L-cysteine, suggesting a causative role for oxidative stress. The results of this study demonstrate that HIF1 $\alpha$ overexpression in ADSCs restored MAEC VEGFR2 function and reduced high glucose-induced MAEC dysfunction [33].

ADSCs participate in various aspects of the wound healing process, including enhancing epidermal cell growth, 


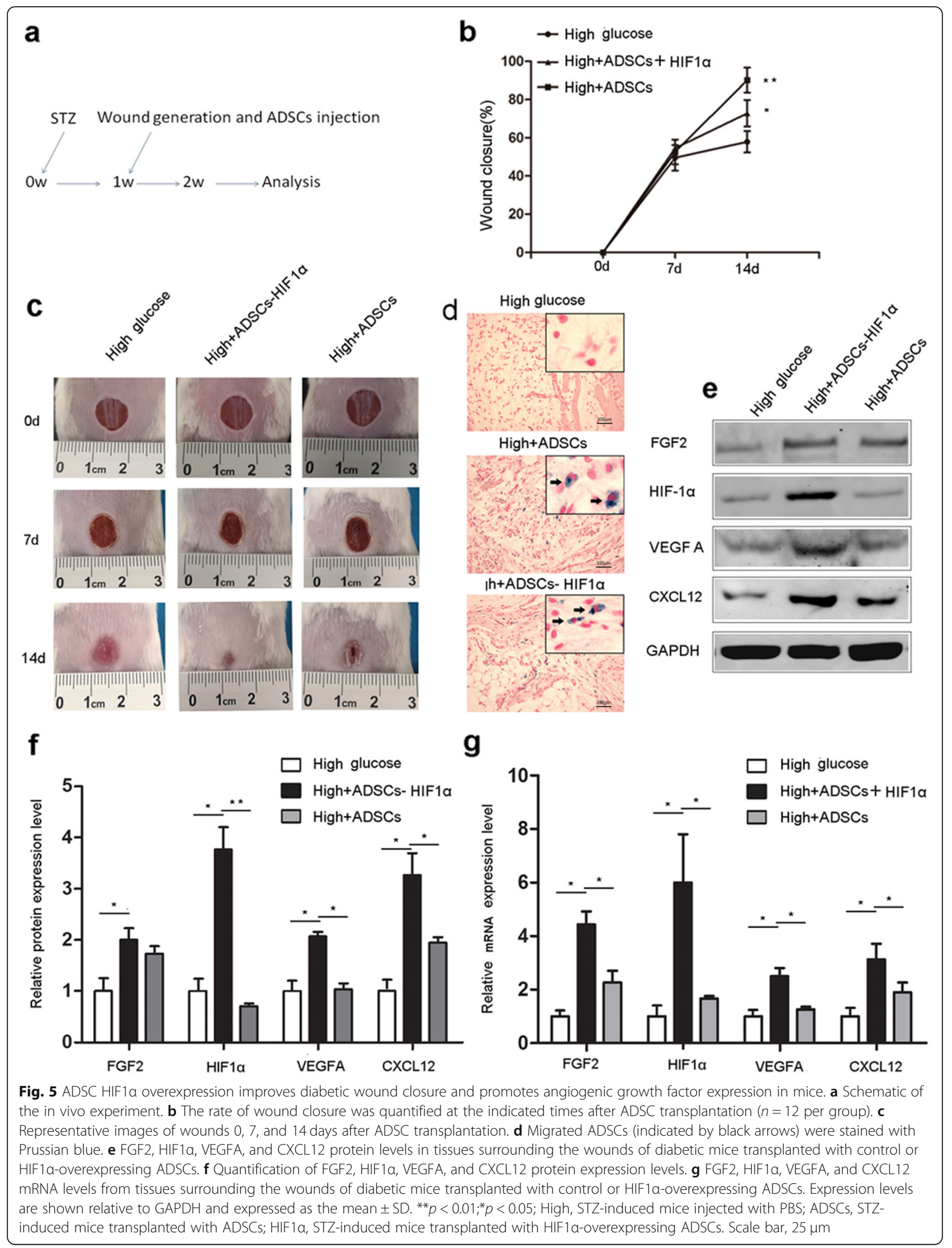




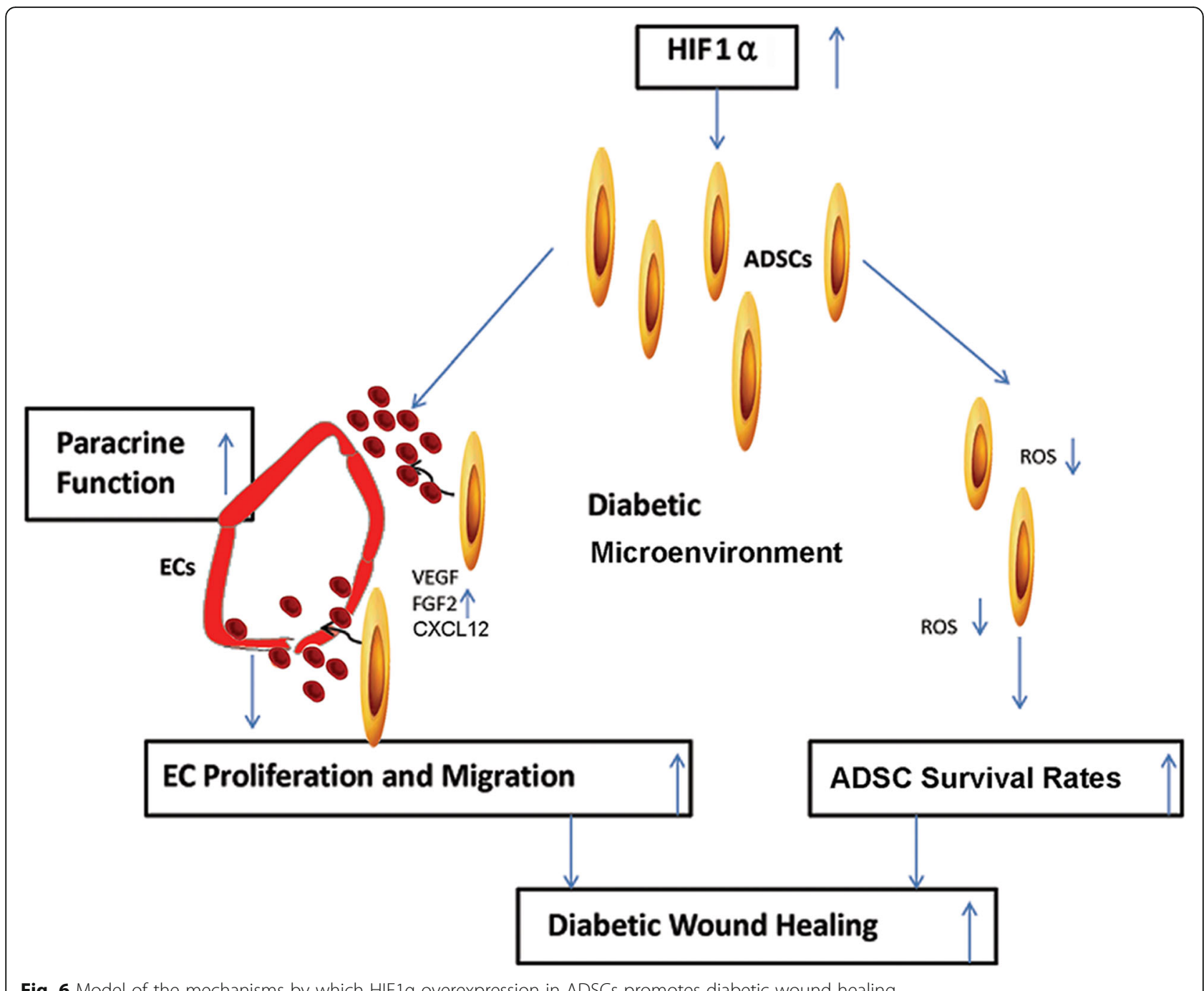

Fig. 6 Model of the mechanisms by which HIF1a overexpression in ADSCs promotes diabetic wound healing

angiogenesis, collagen deposition, anti-inflammatory effects, and wound closure [34, 35]. However, the therapeutic effects of ADSCs are limited by poor survival after transplantation [36]. This problem is even more severe in diabetic wound healing, as high glucose induces ROS generation, resulting in DNA damage and subsequent apoptosis [37]. Thus, increasing the antioxidant defenses of ADSCs is crucial to improve their survival and enable successful ADSC-based therapy for diabetic wound healing. This study indicates that HIF1 $\alpha$ overexpression in ADSCs promotes cell survival under high glucose and low oxygen by stimulating the production of angiogenic growth factors, repressing ROS generation, and preventing DNA damage, resulting in accelerated diabetic wound healing.

Wound healing is a complex process that involves wound contraction, and a limitation of this study is that we did not examine the effects of wound contraction on healing in our in vivo model. In future experiments, it will be important to use splints to fix the mouse skin to prevent skin wound contraction before assaying the effects of ADSC HIF1 $\alpha$ overexpression on wound healing.

\section{Conclusions}

The data indicates that HIF1 $\alpha$ overexpression in ADSCs enhances diabetic wound healing by promoting their paracrine function and preventing DNA damage caused by ROS production, increasing their survival. These beneficial effects significantly accelerated wound closure in diabetic mice transplanted with HIF1 $\alpha$-overexpressing ADSCs. These findings suggest that transplantation of HIF1 $\alpha$ overexpressing ADSCs into injury sites is a promising novel therapeutic strategy for diabetic wound healing, which could also be used in combination with other ADSC-based treatments. We propose that the transplantation of HIF1 $\alpha$ overexpressing ADSCs is an effective and safe alternative to proangiogenic cell transplantation in the treatment of diabetic wounds. 


\section{Abbreviations}

ADSCs: Adipose-derived stem cells; DCFH-DA: 2,7-Dichlorodihydrofluorescein diacetate; EC: Endothelial cells; FGF2: Fibroblast growth factor 2; MAECs: Mouse aortic endothelial cells; PBS: Phosphate-buffered saline; 8OHdG: 8-Hydroxydeoxyguanosine; ROS: Reactive oxygen species; CXCL12: C$\mathrm{X}-\mathrm{C}$ motif chemokine ligand 12; SPIONs: Superparamagnetic polyethyleneimine-coated iron oxide nanoparticles; VEGFA: Vascular endothelial growth factor A; VEGFR2: Vascular endothelial growth factor receptor 2

\section{Acknowledgements}

Not applicable.

\section{Authors' contributions}

$Z W, J X$, and $Y Z$ contributed to the experimental conception and design, performed the experiments, data collection, data analysis, and interpretation, and wrote the manuscript. FZ performed the animal experiments and associated data collection. XYL contributed to the data analysis and interpretation. All authors read and approved the final manuscript.

\section{Funding}

This work was supported by the National Natural Science Foundation of China (81601692) and partly supported by the Program of the Education Department of Liaoning Province (LK2016002), 345 Talent Project of Shengjing hospital of China Medical University.

\section{Availability of data and materials}

The datasets generated and/or analyzed during the current study are included within the article and are available from the corresponding author on reasonable request.

\section{Ethics approval and consent to participate}

All animal procedures were approved by the Medical Ethics Committee of Shengjing Hospital, China Medical University, under the code 2016PS177K. The study was conducted according to the Guide for the Care and Use of Laboratory Animals.

\section{Consent for publication}

Not applicable.

\section{Competing interests}

The authors declare that they have no competing interests.

\section{Author details}

${ }^{1}$ Department of Surgery, Shengjing Hospital of China Medical University, No. 36 Sanhao Street, Heping District, Shenyang 110004, China. ${ }^{2}$ Department of Obstetrics and Gynecology, Reproductive Medicine Center, Shengjing Hospital of China Medical University, No. 36 Sanhao Street, Heping District, Shenyang 110004, China. ${ }^{3}$ Department of Stem Cells and Regenerative Medicine, Shenyang Key Laboratory for Stem Cells and Regenerative Medicine, Key Laboratory of Cell Biology, Ministry of Public Health, and Key Laboratory of Medical Cell Biology, Ministry of Education, China Medical University, No. 77 Puhe Street, Shenbei New District, Shenyang 110122, China. ${ }^{4}$ Department of Pathology, Shengjing Hospital of China Medical University, No. 36 Sanhao Street, Heping District, Shenyang 110004, China.

\section{Received: 14 September 2019 Revised: 11 March 2020}

\section{Accepted: 17 March 2020 Published online: 05 April 2020}

\section{References}

1. Haddad JA, Haddad AN. The past decade in type 2 diabetes and future challenges. Hormones (Athens). 2018;17(4):451-9.

2. Davis FM, Kimball A, Boniakowski A, et al. Dysfunctional wound healing in diabetic foot ulcers: new crossroads. Current Diabetes Reports. 2018;18(1):2.

3. Tao SC, Rui BY, Wang QY, et al. Extracellular vesicle-mimetic nanovesicles transport LncRNA-H19 as competing endogenous RNA for the treatment of diabetic wounds. Drug Delivery. 2018;25(1):241-55.

4. Chalmers J, Joshi R, Patel A. Advances in reducing the burden of vascular disease in type 2 diabetes. Clin Exper Pharmacol Physiol. 2010;35(4):434-7.

5. Demerdash FE, Refaie W, Allakany R, et al. Diabetic retinopathy: a predictor of coronary artery disease. Egyptian Heart J. 2012;64(2):63-8.
6. Bakker W, Eringa EC, Sipkema P, et al. Endothelial dysfunction and diabetes: roles of hyperglycemia, impaired insulin signaling and obesity. Cell Tissue Res. 2005;335(1):165-89.

7. Laing T, Hanson R, Chan F, et al. The role of endothelial dysfunction in the pathogenesis of impaired diabetic wound healing: a novel therapeutic target? Med Hypotheses. 2007;69(5):1029-31.

8. Merkovska L, Jedlickova L, Fedacko JAN, et al. The possible predictive role of laboratory biomarkers for endothelial dysfunction in patients with metabolic syndrome. Atherosclerosis. 2014;235(2):e107.

9. Gimble JM, Katz AJ, Bunnell BA. Adipose-derived stem cells for regenerative medicine. Circ Res. 2007;100(9):1249-60.

10. Gugerell A, Neumann A, Kober J, et al. Adipose-derived stem cells cultivated on electrospun I-lactide/glycolide copolymer fleece and gelatin hydrogels under flow conditions - aiming physiological reality in hypodermis tissue engineering. Burns J Int Soc Burn Injuries. 2015;41(1):163-71.

11. Wang Z, Li H, Zhang D, et al. Effect of advanced glycosylation end products on apoptosis in human adipose tissue-derived stem cells in vitro. Cell Biosci. 2015;5(1):3.

12. Zhe W, Li H, Ran G, et al. Antioxidants inhibit advanced glycosylation endproduct-induced apoptosis by downregulation of miR-223 in human adipose tissue-derived stem cells. Sci Rep. 2016;6:23021.

13. Mahon PC, Hirota K, Semenza GL. FlH-1: a novel protein that interacts with HIF-1 $a$ and VHL to mediate repression of HIF-1 transcriptional activity. Genes Dev. 2001;15(20):2675-86.

14. Wan $\mathrm{XH}, \mathrm{Hu}$ MS, Esquivel $\mathrm{M}$, et al. The role of hypoxia-inducible factor in wound healing. Adv Wound Care. 2014;3(5):390-9.

15. Pore N, Jiang Z, Gupta A, et al. EGFR tyrosine kinase inhibitors decrease VEGF expression by both hypoxia-inducible factor (HIF)-1-independent and HIF-1-dependent mechanisms. Cancer Res. 2006;66(6):3197.

16. Taha MF, Hedayati V. Isolation, identification and multipotential differentiation of mouse adipose tissue-derived stem cells. Tissue Cell. 2010:42(4):211-6.

17. Lin CC, Huang CY, Mong MC, et al. Antiangiogenic potential of three triterpenic acids in human liver cancer cells. J Agric Food Chem. 2011; 59(2):755-62.

18. Christenson LK, Stouffer RL, Strauss JF. Quantitative analysis of the hormoneinduced Hyperacetylation of histone $\mathrm{H} 3$ associated with the steroidogenic acute regulatory protein gene promoter. J Biol Chem. 2001:276(29):27392-9.

19. Wendan Y, Changzhu J, Xuhong S, et al. BMSCs interactions with adventitial fibroblasts display smooth muscle cell lineage potential in differentiation and migration that contributes to neointimal formation. Stem Cells Int. 2016:2016:1-10.

20. Yuan X, Zhang B, Chen N, et al. Isoliquiritigenin treatment induces apoptosis by increasing intracellular ROS levels in HeLa cells. J Asian Nat Prod Res. 2012:14(8):789-98.

21. Liu $X$, Wang $Z$, Wang $R$, et al. Direct comparison of the potency of human mesenchymal stem cells derived from amnion tissue, bone marrow and adipose tissue at inducing dermal fibroblast responses to cutaneous wounds. Int J Mol Med. 2013:31(2):407-15.

22. Yan J, Tie G, Wang S, et al. Diabetes impairs wound healing by Dnmt1dependent dysregulation of hematopoietic stem cells differentiation towards macrophages. Nature Commun. 2018;9(1):33.

23. Galkowska H, Wojewodzka U, Olszewski WL. Chemokines, cytokines, and growth factors in keratinocytes and dermal endothelial cells in the margin of chronic diabetic foot ulcers. Wound Repair Regeneration. 2010; 14(5):558-65.

24. Nie C, Yang D, Xu J, et al. Locally administered adipose-derived stem cells accelerate wound healing through differentiation and vasculogenesis. Cell Transplant. 2011;20(2):205-16.

25. Stubbs SL, Hsiao TF, Peshavariya HM, et al. Hypoxic preconditioning enhances survival of human adipose-derived stem cells and conditions endothelial cells in vitro. Stem Cells Dev. 2012;21(11):1887-96.

26. Botusan IR, Sunkari VG, Savo O, et al. Stabilization of HIF-1alpha is critical to improve wound healing in diabetic mice. Proc Natl Acad Sci U S A. 2008; 105(49):19426-31.

27. Tang Q, Wu H, Lei J, et al. HIF1a deletion facilitates adipose stem cells to repair renal fibrosis in diabetic mice. In Vitro Cell Dev Biol Anim. 2018:54:272-85.

28. Sunkari VG, Lind $F$, Botusan IR, et al. Hyperbaric oxygen therapy activates hypoxia-inducible factor 1 (HIF-1), which contributes to improved wound healing in diabetic mice. Wound Repair Regen. 2015;23(1):98-103. 
29. Biswas S, Roy S, Banerjee J, et al. Hypoxia inducible microRNA 210 attenuates keratinocyte proliferation and impairs closure in a murine model of ischemic wounds. Proc Natl Acad Sci. 2010;107(15):6976-81.

30. Botusan IR, Sunkari VG, Savu O, et al. Stabilization of HIF-1a is critical to improve wound healing in diabetic mice. Proc Natl Acad Sci. 2008;105(49): 19426-31.

31. Wang $H$, Feng $Y$, Jin $X$, et al. Augmentation of hypoxia-inducible factor-1alpha in reinfused blood cells enhances diabetic ischemic wound closure in mice. Oncotarget. 2017:8(69):114251-8.

32. Chamorro-Jorganes A, Araldi E, Penalva LO, Sandhu D, Fernández-Hernando C, Suárez Y. MicroRNA-16 and MicroRNA-424 regulate cell-autonomous angiogenic functions in endothelial cells via targeting VEGFR2 and FGFR1. Arterioscler Thromb Vasc Biol. 2011;31(11):2595.

33. Warren CM, Ziyad S, Briot A, et al. A ligand-independent VEGFR2 signaling pathway limits angiogenic responses in diabetes. Science Signaling. 2014; 7(307):ra1.

34. Ebrahimian T, Pouzoulet FC, Buard V, et al. Cell therapy based on adipose tissue-derived stromal cells promotes physiological and pathological wound healing. Arterioscler Thromb Vasc Biol. 2009;29(4):503-10.

35. Daskalopoulos EP, Janssen BJA, Blankesteijn WM. Targeting Wnt signaling to improve wound healing after myocardial infarction. Methods Mol Biol. 2013; 1037:355-80.

36. Zhu P, Liu J, Shi J, et al. Melatonin protects ADSCs from ROS and enhances their therapeutic potency in a rat model of myocardial infarction. J Cell Mol Med. 2015;19(9):2232-43.

37. Liu JS, Chen Y, Wang H, et al. CRT-112 exendin-4 improves the survival and therapeutic efficacy of implanted stem cells following myocardial infarction. Jacc Cardiovascular Interventions. 2013;6(2):S36.

\section{Publisher's Note}

Springer Nature remains neutral with regard to jurisdictional claims in published maps and institutional affiliations.

Ready to submit your research? Choose BMC and benefit from:

- fast, convenient online submission

- thorough peer review by experienced researchers in your field

- rapid publication on acceptance

- support for research data, including large and complex data types

- gold Open Access which fosters wider collaboration and increased citations

- maximum visibility for your research: over $100 \mathrm{M}$ website views per year

At $\mathrm{BMC}$, research is always in progress.

Learn more biomedcentral.com/submissions 